\title{
Genetic Epidemiology of Fecal Egg Excretion During Schistosoma mansoni Infection in an Endemic Area in Minas Gerais, Brazil
}

\section{J Bethony/*, A Gazzinelli**/+ , A Lopes**, W Pereira**, LF Alves-Oliveira***, S Willams-Blangero*, J Blangero*, PT LoVerde****, R Corrêa-Oliveira}

\author{
Centro de Pesquisas, Rene Rachou-Fiocruz, Belo Horizonte, MG, Brasil *Department of Genetics, Southwest \\ Foundation for Biomedical Research, San Antonio, Texas, USA **Escola de Enfermagem, Universidade Federal \\ de Minas Gerais, Av. Alfredo Balena 190, 30130-100 Belo Horizonte, MG, Brasil ***Cenbios, Universidade Vale \\ do Rio Doce, Governador Valadares, MG, Brasil ****Department of Microbiology, State University of New \\ York at Buffalo, NY, USA
}

There is considerable variation in the level of fecal egg excretion during Schistosoma mansoni infections. Within a single endemic area, the distribution of egg counts is typically overdispersed, with the majority of eggs excreted coming from a minority of residents. The purpose of this study was to quantify the influence of genetic factors on patterns of fecal egg excretion in a rural study sample in Brazil. Individual fecal egg excretions, expressed in eggs per gram of feces, were determined by the Kato-Katz method on stool samples collected on three different days. Detailed genealogic information was gathered at the time of sampling, which allowed assignment of 461 individuals to 14 pedigrees containing between 3 and 422 individuals. Using a maximum likelihood variance decomposition approach, we performed quantitative genetic analyses to determine if genetic factors could partially account for the observed pattern of fecal egg excretion. The quantitative genetic analysis indicated that between 21-37\% of the variation in $\mathrm{S}$. mansoni egg counts was attributable to additive genetic factors and that shared environment, as assessed by common household, accounted for a further 12$21 \%$ of the observed variation. A maximum likelihood heritability $\left(\mathrm{h}^{2}\right)$ estimate of $0.44 \pm 0.14$ (mean \pm $S E$ ) was found for the 9,604 second-and higher-degree pairwise relationships in the study sample, which is consistent with the upper limit (37\%) of the genetic factor determined in the variance decomposition analysis. These analyses point to the significant influence of additive host genes on the pattern of S. mansoni fecal egg excretion in this endemic area.

Key words: Schistosoma mansoni - genetic factors - household factors - Minas Gerais - Brasil

There is considerable variation in the level of fecal egg excretion in schistosome infections. In studies from Nigeria (Pugh \& Gilles 1978), Ethiopia

Grant support by NIH funded International Center for Infectious Disease Research (U01 AI45451), Programa de Apoio a Grupos de Excelência, Conselho de Desenvolvimento Tecnológico e Científico-CNPq and Fundação de Amparo à Pesquisa do Estado de Minas Gerais. J Bethony is supported by an International Research Scientist Development Award, from the Fogarty International Center, National Institutes of Health, USA. Informed consent was obtained from all subjects and their parents. The human subject studies were approved by the Ministry of Health of Brazil and the IRB of the State University of New York at Buffalo.

+Corresponding author. Fax: +55-31-3286.8801. E-mail: andreag@enf.ufmg.br

Received 14 May 2001

Accepted 25 July 2001
(Polderman 1979), Ghana (Scott et al. 1982), and Zaire (Polderman et al. 1985a, b), wide variation in fecal egg counts has been observed between nearby endemic areas. Within a single endemic area, variation in fecal egg excretion levels has been noted among individuals and between households (Bradley \& May 1978). Analysis of fecal egg excretion from individuals resident in single endemic areas often show that the distribution of egg counts is overdispersed (Bradley \& May 1978) with marked skewness and leptokurtosis. An important consequence of the overdispersed distribution in egg counts is that the majority of eggs excreted in a community come from a minority of residents. Results of a study conducted in Ethiopia indicated that $50 \%$ of the eggs excreted came from $5 \%$ of the community (Polderman 1979), and in a Brazilian study the same proportion came from $6 \%$ of the community (Lima e Costa et al. 1985). Day to day fluctuations in egg counts for a single individual have also been documented (Engels et al.1996). 
Numerous factors are thought to influence levels of egg excretion. These include the presence or absence of habitats suitable for intermediate hosts (Goll et al. 1984), patterns of human activity, socioeconomic status (Lima e Costa et al. 1985), densitydependent functions (Medley \& Anderson 1985), and the immune response of the host (Doenhoff et al. 1978, Damian 1987, Ngaiza \& Doenhoff 1990, Amiri et al. 1992). Recently, a relationship between genetic factors and infection intensity has been documented. Using data from 269 families, Abel et al. (1991) detected a codominant major gene (SM1) accounting for a substantial proportion of the variation in infection intensity among individuals resident in a single endemic area. Marquet et al. (1996) localized the SM1 gene to chromosome 5q31-q33, an area that contains various interleukin (IL) genes (e.g., IL-4, IL-5, and IL-13). While these genetic studies explicitly test for the influence of a major single locus, we have used a simple quantitative variance decomposition model to partition the observed variance in fecal egg excretion into components attributable to additive genetic (polygenes), shared environmental (household), and random environmental factors. Our analytical focus has been on the correlation and heritability of fecal egg excretion among relatives in extended multi-household pedigrees. Herein, we demonstrate the role of additive genetic and shared environmental factors on the variation in fecal egg excretion in a single endemic community in Brazil.

\section{POPULATION, MATERIALS AND METHODS}

Study sample - The study population was composed of 461 inhabitants, ranging in age from 5 to 85 years, of Córrego de Melquíades (Governador Valadares municipality), an area that is endemic for Schistosoma mansoni and is situated in the State of Minas Gerais, Brazil. Individuals were sampled through house-to-house surveys. Genealogical and demographic information including age, sex, parental names, and residences for all individuals were collected. These data allowed construction of extended multi-household pedigrees using PEDSYS, a pedigree-based data management system (Dyke 1989). Individuals were defined as belonging to the same pedigree if they were biologically related to anyone else in the pedigree or if they were unrelated to anyone else in the pedigree who had not yet had children, but lived in the same house as their spouse, who was biologically related to someone else in the pedigree. The unrelated spouses were included primarily to improve estimation of common household effects. The 461 individuals belonged to 14 pedigrees, ranging in size from 3 to 422 individuals, (Table I) and were distributed over 113 different households (Table II). Seven unre- lated single individuals were retained in the analysis as independents. Table II presents the numbers of individuals (1-12) sampled per household. Fiftytwo $(46 \%)$ of the households were represented by between 1 and 3 individuals, while sixty-one (54\%) of the households were represented by four or more individuals.

TABLE I

Distribution of sampled individuals by family size

\begin{tabular}{ccc}
\hline $\begin{array}{l}\text { Pedigree } \\
\text { size }\end{array}$ & $\begin{array}{c}\text { No. of } \\
\text { pedigrees }\end{array}$ & $\begin{array}{c}\text { No. of sampled } \\
\text { individuals }\end{array}$ \\
\hline 1 & 7 & 7 \\
3 & 2 & 6 \\
4 & 1 & 4 \\
6 & 2 & 12 \\
10 & 1 & 10 \\
422 & 1 & 422 \\
\hline Total & 14 & 461 \\
\hline
\end{tabular}

TABLE II

Number of household representatives in the pedigree sample

\begin{tabular}{lcc}
\hline $\begin{array}{l}\text { No. of household } \\
\text { representatives }\end{array}$ & $\begin{array}{c}\text { No. of } \\
\text { households }\end{array}$ & $\begin{array}{c}\text { No. of sampled } \\
\text { individuals }\end{array}$ \\
\hline 1 & 13 & 13 \\
2 & 17 & 34 \\
3 & 22 & 66 \\
4 & 19 & 76 \\
5 & 17 & 85 \\
6 & 10 & 60 \\
7 & 5 & 35 \\
8 & 4 & 32 \\
9 & 3 & 27 \\
10 & 1 & 10 \\
11 & 1 & 11 \\
12 & 1 & 12 \\
\hline Total & 113 & 461 \\
\hline
\end{tabular}

Parasitological data - Infection intensities were measured by individual fecal egg excretions, expressed in eggs per gram of feces (epg), that were the arithmetic mean of at least three determinations performed by the Kato Katz method (Katz \& Pellegrino 1972) on stool samples collected on three different days. To reduce heteroscedasticity, we performed all analyses on the log transformed variable (epg + 1). Prevalence of infection was determined as a score of $\geq 1 \mathrm{epg}$. A survey conducted at the time of fecal collection indicated that none of the individuals in the study sample had ever received schistosomicidal treatment. 
Analytical methods - Variance components analysis was used to decompose the variance of a given phenotype into its constituent sources (Hopper \& Mathews 1982, Lange \& Boehnke 1983). For the present study, we were interested in obtaining the relative importance of genes, shared household, and random environment on the variation in fecal egg excretion in schistosome infections. To formally test for the involvement of both genes and household, we used a method that allowed the use of all of our pedigree and household sharing information simultaneously. We used the pedigree based maximum likelihood variance decomposition method as implemented in the computer program SOLAR (Almasy 1998) to assess the relative importance of genetic factors in the determination of the variation of fecal egg counts. By assuming multivariate normality as a working model within pedigrees, the likelihood of any pedigree can be easily specified and numerical procedures can be used to estimate the variance component parameters (Beaty et al. 1985). Likelihood estimation assuming multivariate normality yields consistent parameter estimates, even when the distributional assumptions are violated. Standard likelihood statistics tests were used for assessing the statistical significance of focal parameters. Hypothesis testing was performed by constraining the specific variance component parameters $\left(h^{2}\right.$ and $\left.c^{2}\right)$ to 0 and seeing whether the constrained model showed a significantly worse fit than the unconstrained model. Covariate effects were also tested using this procedure.

For the evaluation of specific familial correlations, maximum likelihood techniques, as implemented in FISHER (Lange et al. 1988), were used to choose the estimate of the correlation coefficient that provided the greatest likelihood of observing the data. This approach used the complete pedigree information and allowed for the simultaneous estimation of covariate effects. To determine whether residence in the same household increased the correlation among individuals with regard to fecal egg excretion we stratified these correlations by household sharing status (i.e., whether the pair of individuals lived in the same or different households at the time of sampling). If unrelated pairs who share a household have a higher correlation than unrelated individuals living in different households, we have presumptive evidence of the role of shared exposure. In contrast, if the correlation between relatives living in the same household is higher than that between unrelated individuals living in the same household, we have evidence for a genetic contribution to the level of infection intensity.

\section{RESULTS}

Sample prevalence, intensity of infection, covariate effects - Quantitative egg counts (Katz \& Pellegrino 1972) were performed on 461 individuals. Fig. 1 shows the relationship between prevalence of infection and ten-year age intervals, with the bars indicating the $95 \%$ confidence intervals for the prevalence estimate for each interval. The total prevalence was $62 \%(n=284)$. The maximum increase in prevalence was observed between the 5-10 (47\%) and 11-19 (78\%) age intervals. Fig. 2 depicts the relationship between log transformed epg and ten-year age intervals, with the bars indicating the $95 \%$ confidence intervals for the mean egg count for each ten-year age interval. The mean $\log$ transformed epg for the study sample was 106 $(95 \% \mathrm{CI}=82,131)$. The highest egg counts were observed in the 10-19 year age interval, with a mean epg of $141(95 \% \mathrm{CI}=99,183)$. The maximum increase in fecal egg count occurred between the 1-9 year (mean epg $=92 \pm 67$ ) and the 10-19 year (mean epg $=141 \pm 43$ ) age intervals.

We simultaneously allowed for sex and age effects in the analysis. There was no significant effect of age on fecal egg excretion levels. There was a significant effect of sex $(\mathrm{P}<0.00001)$, with $2 \%$ percent of the variation explained by this covariate, with males having higher egg per gram of feces than females.

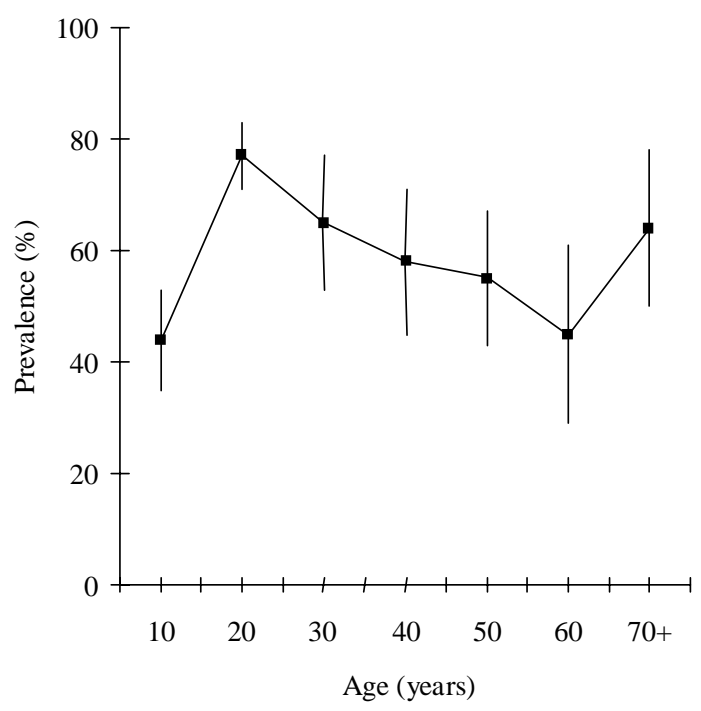

Fig. 1: the relationship between prevalence of infection with Schistosoma mansoni and ten year age intervals from Melquíades, Minas Gerais, Brazil June 1997. Bars indicate 95\% confidence intervals. 


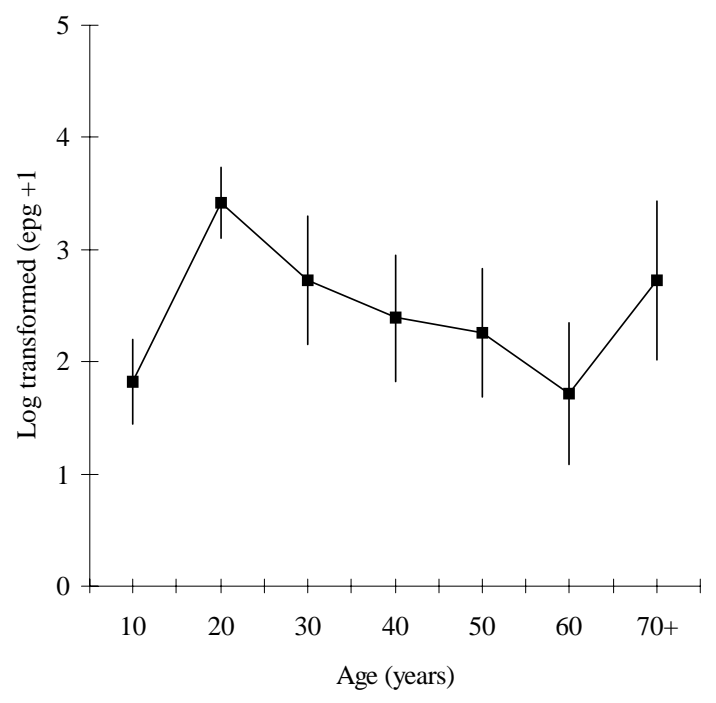

Fig. 2: the relationship between egg excretion in Schistosoma mansoni infection, measured as log transformed eggs per gram of feces $(e p g+1)$, and ten year age intervals from Melquíades, Minas Gerais, Brazil June 1997. Bars indicate 95\% confidence intervals.

Variance components analysis - Table III shows the results of the variance components analyses. Four possible variance component models are presented. The two variance components considered are the relative genetic contribution (i.e., heritability, $\left.h^{2}\right)$ and the shared household component $\left(c^{2}\right)$. The zeros appearing in the parenthesis $(0)$ indicate constraints on the model. The general model, which allows for both genetic and shared household effects, showed a heritability of $0.21 \pm 0.14$ and a common household effect of $.12 \pm 0.08$. This indicates that $21 \%$ of the variation in egg counts may be due to genetics and $12 \%$ due to shared household environment. The remaining $67 \%$ is attributed to random environmental effects. Each of the following three submodels were then compared to the general model: (1) the genetic model, (2) the household model, and (3) the sporadic model. In the genetic model, where only the genetic component was estimated, a heritability of $0.37 \pm .08$ was found for the variation in fecal egg excretion. In the household model, a shared environmental effect of $0.22 \pm$ 0.05 was observed. The sporadic model, where schistosome fecal egg excretion was tested for random distribution, was clearly rejected $(p=0.0001)$, which indicates that the clustering of fecal egg excretion is not random.

Since neither the genetic ( $p=0.064)$ nor the household model $(p=0.053)$ was significantly different from the general model, the results of the variance component analysis can point only to a range of possible effects. Thus, additive genes may account for between $21-37 \%$ of variance in fecal egg excretion; i.e., nearly $40 \%$ of the variability in fecal egg excretion may be due to genetic factors. Similarly, the estimate between $12 \%$ and $22 \%$ for the household effects indicates that up to an additional quarter of the variance may be due to shared environment.

Correlations among relatives - In order to further understand the estimates observed above, we utilized the extensive pairwise relationships in the study sample to determine familial correlations. Table IV shows the pairs of relatives in the study sample by degree of relatedness. There are over 10,000 relative pairs for genetic analysis, with over 9,000 second degree or higher relative pairs. The use of these pairwise relationships markedly increased our power to detect genetic factors because of (a) the large number of such pairs and (b) the ability to stratify the pairs based on "shared" or "different" residence. Table V presents the correlations between various classes of relatives stratified by residence. The maximum likelihood estimates of the correlations are provided along with the standard error and the number of pairs that contributed the information. Among first degree relatives the presence of genetic factors is confirmed by the correlation of $0.17 \pm 0.07$ for parent offspring pairs from shared households and $0.31 \pm 0.08$ for sibling pairs from shared households. Both of the above correlations were higher than the correlation of 0.11

TABLE III

Variance decomposition of intensity of infection with Schistosoma mansoni: maximum likelihood estimates and robust test scores

\begin{tabular}{lcccc}
\hline & \multicolumn{2}{c}{ Source of variation } & & \\
\cline { 2 - 4 } Model & Genetic & Household & Test Score & $P$ \\
\hline General & $0.205 \pm 0.14$ & $0.117 \pm .08$ & - & - \\
Genetic & $0.373 \pm 0.08$ & $(0)$ & 2.32 & .064 \\
Household & $(0)$ & $0.223 \pm 0.05$ & 2.62 & .053 \\
Sporadic & $(0)$ & $(0)$ & 42.78 & $<.0001$ \\
\hline
\end{tabular}

Each model is tested against the general model; — : indicates does not apply 
TABLE IV

Relative pairs in the study sample

\begin{tabular}{lc}
\hline Relative pairs & Number of pairs \\
\hline Parent-offspring & 542 \\
Siblings & 688 \\
Second degree & 929 \\
Third degree & 1,525 \\
Fourth degree & 1,568 \\
Fifth degree & 1,832 \\
Sixth degree & 1,499 \\
Seventh degree & 1,207 \\
Eighth degree & 782 \\
Ninth degree & 252 \\
Tenth degree & 10 \\
\hline Total & 10,834
\end{tabular}

\pm 0.09 for spouses, which is an indication of the presence of shared household effects as spouses are typically unrelated but share a similar residence. Evidence for genetic effects can also be found in the correlations which remained after the relative pairs were stratified for residence in different households, with a parent-offspring correlation of $0.09 \pm$ 0.11 and a sibling correlation of $0.14 \pm 0.10$. Among the 9,604 second degree and higher relative pairs, the genetic component in fecal egg excretion was demonstrated by a heritability $\left(h^{2}\right)$ estimated at 0.44 \pm 0.14 . The strength of this estimate derives from the fact that higher order relative pairs seldom live together, which avoids the potential confusion of household and genetic effects. The estimate clearly confirms the upper limit of the variance component analysis in which $44 \%$ of the variance in fecal egg excretion was attributed to genetic factors.

\section{DISCUSSION}

The size and complexity of the extended multihousehold pedigrees in the study sample enabled us to examine the additive effect of host genes on fecal egg excretion in S. mansoni infection. The distribution of fecal egg counts in the study sample was similar to other areas endemic for $S$. mansoni or S. haematobium in Africa (Forsyth \& Bradley
1966, Pugh \& Gilles 1978, Polderman 1979, Scott et al. 1982, Goll et al. 1984, Polderman et al. 1985a,b) and S. mansoni in Brazil (Lima e Costa et al. 1985). This includes both the convex relationship between age and infection intensity and the overdistribution of egg counts, where a minority of individuals accounted for the majority of eggs excreted (Bradley \& May 1978).

The quantitative genetic analysis indicated that $21-37 \%$ of the variation in $S$. mansoni egg counts in the study sample was attributable to additive genetic factors and that shared household environment accounted for a further $12-21 \%$ of the observed clustering. This was demonstrated in three ways. First, the rejection of the sporadic model $(p<$ 0.0001 ) indicated that the variation in fecal egg excretion was not randomly distributed. Second, the variance components analysis, which partitions the variation in fecal egg counts into genetic, household, and random environmental influences, indicated an additive genetic influence $\left(h^{2}\right)$ of $21-37 \%$ and a shared household influence $\left(c^{2}\right)$ of $12-22 \%$. Third, a heritability $\left(h^{2}\right)$ estimate based only on the numerous second degree and higher pairwise relationships in the study sample, indicated an additive genetic effect of $44 \%$ for fecal egg counts. The strength of the latter estimate was derived from the fact that second degree and higher relative pairs (e.g., first cousins, second cousins, grandparentsgrandchildren, etc.) seldom live together, which avoids the potential overlap of household and genetic effects. Furthermore, the heritability estimate from second degree and higher relatives is consistent with the upper limit (37\%) of the genetic factor $\left(h^{2}\right)$ determined by the variance components analysis. Together, these analyses point to the significant influence of additive host genes on the distribution of fecal egg excretion in this endemic area.

The determinants of fecal egg excretion are still fundamentally debated (Gryseels \& de Vlas 1996). Previous studies have emphasized the relationship between age and egg per gram of feces in S. mansoni infection (Woolhouse 1998). There is a substantial literature that attempts to explain the decline in the eggs per gram of feces levels with age as due to (1)

\section{TABLE V}

Correlations for $\ln (\mathrm{epg}+1)$ for relative pairs in the shared and different households

\begin{tabular}{llrr}
\hline Relationship & Household & No. of pairs & $r \pm$ SE \\
\hline Spouses & Shared & 121 & $.11 \pm .09$ \\
Parent-offspring & Shared & 225 & $.17 \pm .07$ \\
Siblings & Shared & 125 & $.31 \pm .08$ \\
Parent-offspring & Different & 78 & $.09 \pm .11$ \\
Siblings & Different & 104 & $.14 \pm .10$ \\
\hline
\end{tabular}

Heritability $\left(h^{2}\right)$ among second degree and higher relatives $0.44 \pm .14$ 
reduced exposure, (2) increased innate resistance, (3) acquired immunity, or (4) a combination of these factors. However, the role of these factors on the relationship between age and infection intensity remains controversial, primarily because exposure to schistosome infection is difficult to assess and the nature of the protective immune response to schistosome infection remains open to debate. Another set of studies has emphasized the role of density-dependent effects on fecal egg excretion. An early notion of density dependence was based on the principle that the per capita fecundity of helminthic parasites is inversely related to the density of worms (Engels et al 1996). This density-dependent fecundity was thought to be due to worm crowding and was, therefore, considered an attribute of the parasite. A conceptually related notion of density dependence incorporates a form of acquired resistance in that additional schistosomes fail to establish once a threshold worm burden is reached as the result of immunological or non-immunological factors (Woolhouse 1998). In this case, density-dependent effects were considered an attribute of the host. In both cases, the role of density-dependent effects on the distribution of fecal egg counts remains controversial and difficult to assess.

Another important pattern in schistosome epidemiology is that fecal egg counts are typically overdispered, with high egg counts aggregated in a minority (5-6\%) of the individuals (Bradley \& May 1978). The possession of high egg counts is a relatively persistent rather than transient attribute. There is also evidence that a subject's tendency to have a high egg count relative to others in the group may persist despite successful chemotherapy (Bradley \& May 1978). The question that presents itself, then, is whether or not the distribution in fecal egg counts in endemic populations reflects innate host factors. The humoral and cellular aspects of the host immune response have been implicated as the innate host factors most likely to influence fecal egg excretion (Damian 1987). Doenhoff et al. (1978) and Doenhoff et al. (1985) noted considerably reduced rates of excretion of $S$. mansoni egg in T-cell-deprived mice, compared to immunologically intact controls; serum transfer with chronic infection serum or egg antigen-immunized serum induced a partial restoration of egg excretion. Recently Karanja et al. (1998) observed that patients who were infected with $S$. mansoni and were seropositive for HIV excreted fewer eggs than individuals who were not seropositive for HIV infection. Furthermore, those in the seropositive group showed a significant correlative relationship between egg excretion ratios and CD4+ lymphocyte percentages. These observations are compatible with the hypothesis that schistosome eggs exit the human host through the requisite facilitation of functional immune responses, and that the efficacy of this process decreases in schistosomiasis patients co-infected with HIV as their peripheral blood $\mathrm{CD} 4+$ cell is lower. The granuloma, which is immune dependent, has also been shown to play an important role in the excretion of schistosome eggs in mice (Doenhoff et al. 1986). Amiri et al. (1992) reported that the immunoregulatory cytokine tumor necrosis factor alpha (TNF- $\alpha$ ) is necessary and sufficient to reconstitute granuloma formation in schistosome-infected SCID mice. Moreover, it was found that the parasitic worms required TNF- $\alpha$ for egg laying and for excretion of eggs from the host. The implication of these results are twofold. The first is that the parasite has adapted so successfully to its host that it may use host-derived immunoregulatory proteins for replication and transmission. The second is that the levels of host immunoregulatory proteins such as TNF- $\alpha$ may be determined by the genetic constitution of the host and, as such, it may be the host which influences the distribution of fecal egg counts in endemic communities. Several recent studies have shown that polymorphisms in the TNF- $\alpha$ gene, located on human chromosome 6 , influence levels of this protein in response to a variety of parasitic infections (refs). Future genetic studies are planned to examine the role of polymorphisms in this gene and others in the current study sample.

Previous studies of the role of host genes in $S$. mansoni infection have generated significant results. In an initial study using 269 individuals belonging to 20 families, Abel et al. (1991) detected a major condominant gene or locus accounting for a substantial portion of the variation in infection intensity. More recently, segregation (MullerMyhsok et al. 1997) and linkage studies (Marquet et al. 1996) from several other groups have narrowed the search to a major locus on human chromosome 5q31-q33 as influencing the intensity of infection. The present study adds to the growing literature on the genetic component of schistosome infection by confirming the role of additive genetic effects on the variation in egg excretion in S. mansoni infection. Additionally, this is the first study to formally evaluate both genetic and shared household effects. The further identification of host genes regulating schistosome-human interactions will provide new ways of understanding the factors critical in controlling schistosome infection and disease development.

\section{ACKNOWLDGEMENTS}

To the families of Córrego de Melquíades for participating in this study. To Ms Marluce Rodrigues Lima, 
Ms Maria de Fátima da Silva, and Ms Lillian Cardoso Moreira for assistance throughout the study. To the American Airlines for facilitating transport of supplies from Buffalo, NY, to Belo Horizonte, Brazil.

\section{REFERENCES}

Abel L, Demenais F, Prata A, Souza AE, Dessein A 1991. Evidence for the segregation of a major gene in human susceptibility/resistance to infection by Schistosoma mansoni. Am J Hum Genet 48: 959-970.

Almasy LBJ 1998. Multipoint quantitative trait linkage analysis in general pedigrees. Am J Hum Genet 62: 1198-1211.

Amiri P, Locksley RM, Parslow TG, Sadick M, Rector E, Ritter D, McKerrow JH 1992. Tumour necrosis factor alpha restores granulomas and induces parasite egg-laying in schistosome-infected SCID mice. Nature 356: 604-607.

Beaty TH, Self SG, Liang KY, Connolly MA, Chase GA, Kwiterovich PO 1985. Use of robust variance components models to analyse triglyceride data in families. Ann Hum Genet 49: 315-328.

Bradley DJ, May RM 1978. Consequences of helminth aggregation for the dynamics of schistosomiasis. Trans $R$ Soc Trop Med Hyg 72: 262-273.

Damian RT 1987. The exploitation of host immune responses by parasites. J Parasitol 73: 3-13.

Doenhoff M, Musallam R, Bain J, McGregor A 1978. Studies on the host-parasite relationship in Schistosoma mansoni infected mice: the immunological dependence of parasite egg excretion. Immunology 35 : 771-778.

Doenhoff MJ, Hassounah OA, Lucas SB 1985. Does the immunopathology induced by schistosome eggs potentiate parasite survival? Immunol Today 6: 203206.

Doenhoff MJ, Hassounah O, Murare H, Bain J, Lucas S 1986. The schistosome egg granuloma: immunopathology in the cause of host protection or parasite survival? Trans R Soc Trop Med Hyg 80: 503-514.

Dyke B 1989. PEDSYS, PGL Tech. Report No. 2, Southwest Foundation for Biomedical Research, San Antonion, TX.

Engels D, Sinzinkayo E, Gryseels B 1996. Day-to-day egg count fluctuation in Schistosoma mansoni infection and its operational implications. Am J Trop Med Hyg 54: 319-324.

Forsyth DM, Bradley DJ 1966. The consequences of Bilharziasis. Medical and public health importance in North-west Tanzania. Bull WHO 34: 715-735.

Goll PH, Wilkins HA, Marshall TF 1984. Dynamics of Schistosoma haematobium infection in a Gambian community. II. The effect on transmission of the control of Bulinus senegalensis by the use of niclosamide. Trans $R$ Soc Trop Med Hyg 78: 222226.

Gryseels B, de Vlas SJ 1996. Worm burdens in schistosome infections. Parasitol Today 12: 115-119.

Hopper JL, Mathews JD 1982. Extensions to multivariate normal models for pedigree analysis. Ann Hum Genet 46: 373-383.
Karanja DM, Boyer AE, Strand M, Colley DG, Nahlen BL, Ouma JH, Secor WE 1998. Studies on schistosomiasis in western Kenya. II. Efficacy of praziquantel for treatment of schistosomiasis in persons coinfected with human immunodeficiency virus-1. Am J Trop Med Hyg 59: 307-311.

Katz N, Pellegrino J 1972. A simple device for quantitative stool thick smear technique in schistosomiasis mansoni. Rev Inst Med Trop São Paulo 14: 397-400.

Lange K, Boehnke M 1983. Extensions to pedigree analysis. IV. Covariance components models for multivariate traits. Am J Med Genet 14: 513-524.

Lange K, Weeks D, Boehnke M 1988. Programs for Pedigree Analysis: MENDEL, FISHER, and GENE. Genet Epidemiol 5: 471-472.

Lima e Costa MF, Rocha RS, de Almeida Magalhaes MH, Katz N 1985. A clinico-epidemiological survey of schistosomiasis mansoni in a hyperendemic area in Minas Gerais State (Comercinho, Brazil). I. Differences in the manifestations of schistosomiasis in the town centre and in the environs. Trans $R$ Soc Trop Med Hyg 79: 539-545.

Marquet S, Abel L, Hillaire D, Dessein H, Kalil J, Feingold J, Weissenbach J, Dessein AJ 1996. Genetic localization of a locus controlling the intensity of infection by Schistosoma mansoni on chromosome 5q31q33. Nat Genet 14: 181-184.

Medley G, Anderson RM 1985. Density-dependent fecundity in Schistosoma mansoni infections in man. Trans R Soc Trop Med Hyg 79: 532-534.

Muller-Myhsok B, Stelma FF, Guisse-Sow F, Muntau B, Thye T, Burchard GD, Gryseels B, Horstmann RD 1997. Further evidence suggesting the presence of a locus, on human chromosome 5q31-q33, influencing the intensity of infection with Schistosoma mansoni. Am J Hum Genet 61: 452-454.

Ngaiza JR, Doenhoff MJ 1990. Blood platelets and schistosome egg excretion. Proc Soc Exp Biol Med 193: 73-79.

Polderman AM 1979. Transmission dynamics of endemic schistosomiasis. Trop Geogr Med 31: 465475.

Polderman AM, Mpamila K, Manshande JP, BouwhuisHoogerwerf ML 1985a. Methodology and interpretation of parasitological surveillance of intestinal schistosomiasis in Maniema, Kivu Province, Zaire. Ann Soc Belg Med Trop 65: 243-249.

Polderman AM, Mpamila K, Manshande JP, Gryseels B, van Schayk O 1985b. Historical, geological and ecological aspects of transmission of intestinal schistosomiasis in Maniema, Kivu Province, Zaire. Ann Soc Belg Med Trop 65: 251-261.

Pugh RN, Gilles HM 1978. Malumfashi Endemic Diseases Research Project, III Urinary Schistosomiasis: a Longitudinal Study. Ann Trop Med Parasitol 72: 471-482.

Scott D, Senker K, England EC 1982. Epidemiology of human Schistosoma haematobium infection around Volta Lake, Ghana, 1973-75. Bull WHO 60: 89-100.

Woolhouse M 1998. Patterns in parasite epidemiology: the peak shift. Parasitol Today 14: 428-434. 
